\title{
A Passively Adaptive Microspine Grapple for Robust, Controllable Perching
}

\author{
Hai-Nguyen Nguyen ${ }^{* 1}$ Robert Siddall ${ }^{* \dagger 1}$, Brett Stephens ${ }^{1}$, Alberto Navarro-Rubio ${ }^{1}$ and Mirko Kovač ${ }^{1,2}$
}

\begin{abstract}
The application of flying systems to practical tasks is consistently limited by the poor endurance of hovering robots. The ability to perch to fixed surfaces allows a robot to gather data and inspect structures in a low power state, while retaining the access and manoeuvrability that flight offers. In this paper we present a passively adaptive perching mechanism which allows an aerial vehicle to stably attach to a variety of surfaces including tree branches and pipelines. This is enabled by a compliant grapple module, which passively conforms to the surface of convex perching targets, ensuring reliable traction and a very high load capacity (tension tested to $>60 \mathrm{~kg}$ in some instances) whilst still releasing effortlessly. This is due to the mechanics of the grapple, which is designed to passively tighten and attach to a variety of branch diameters and shapes. The grapple is paired with a hybrid force-motion controller which allows the cable tension to be regulated as the vehicle achieves the desired attitude. The hybrid control approach exploits the mechanical compliance of the system to ensure reliable, stable attachment to irregular natural structures, and the addition of a winch allows the robot to stably orient itself in any position or orientation relative to the branch. This approach demonstrates tensile perching using adaptive anchors. The presented subsystems can be applied to other robots where high force authority is required.
\end{abstract}

\section{INTRODUCTION}

The high power consumption of propeller-driven hovering flight severely limits the endurance of Unmanned Aerial Vehicles (UAVs) [1]. This typically results in flying times of around 20 minutes, depending on the task and design constraints. In the natural world, perching is often used to remain aloft for long durations and extend flights. For UAVs, perching enables robots to avoid the high energy penalty of flight, perform a wider variety of tasks, and extend sensing time. The challenge is then in ensuring that a robot can robustly and reliably attach and detach. This is particularly challenging where the perch is unknown or has a complex and irregular geometry.

While larger animals (e.g. raptors) typically perch using considerable sensing and control, often exploiting highly nonlinear aerodynamic effects, smaller animals typically exhibit passive mechanical intelligence when perching or interacting with fixed surfaces [2], [3]. The latter approach is particularly useful in small robots, where sensing and computation are limited, and with judicious design a passive attachment can be far more robust, particularly when faced

*These authors contributed equally to this work

†To whom correspondence should be addressed; r.siddall13@imperial.ac.uk

${ }^{1}$ Aerial Robotics Laboratory, Imperial College London, South Kensington, London. Email: h.nguyen@imperial.ac.uk, r.siddall13@imperial.ac.uk, brett.stephens18@imperial.ac.uk, m.kovac@imperial.ac.uk

${ }^{2}$ Materials and Technology Centre of Robotics, Swiss Federal Laboratory for Materials Science and Technology (Empa), Zurich

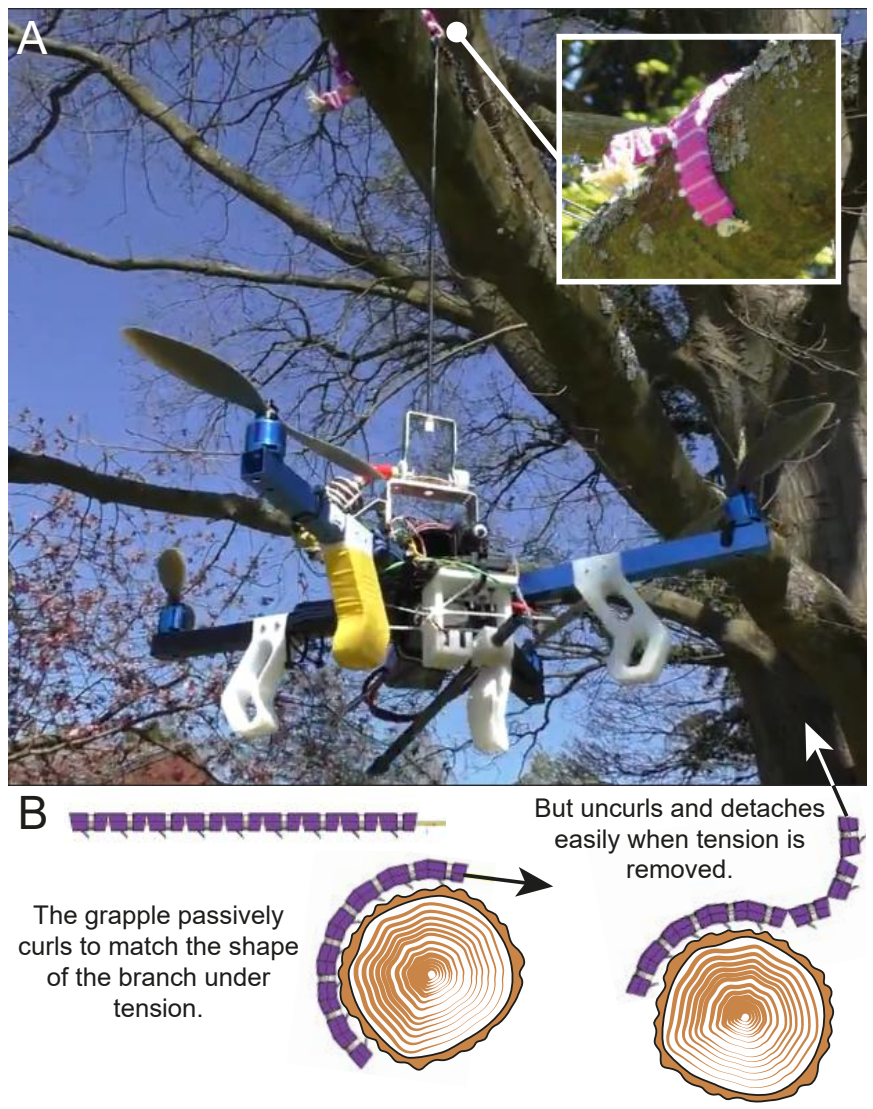

Fig. 1: A: Perching robot after suspending itself from a tree branch. Inset shows the compliant grapple passively wrapped around a branch. B: The compliant grapple passively curls to the shape of the branch, engaging the microspines to share the load, but still detaches easily.

with an unstructured outdoor environment and noisy sensor data.

Examples of passive attachment systems include directional adhesives [4], compliant grippers [5], hooked tethers [6] and microspines [7], [8]. Spines exploit the rugosity of natural surfaces to support contact shear loads, making them most effective at the small scale, where weight can be sufficiently distributed across surface asperities. Microspines have been used in climbing robots [9], mechanisms for perching [10] and grippers [11].

The robot presented in this paper is designed with a passive-adaptive geometry which will wrap itself around objects when tension is applied (figure 1A-B), but which will release easily when tension is removed. The grapple is formed of several rigid links able to transfer contact 
forces, but flexibly connected such that they can adapt to different perch sizes, and making it widely insensitive to variations in perch geometry, improving on previous systems for attachment to tree branches [12], which could not attach to branches above a maximum size.

By placing microspines along the underside of the grapple, a UAV is able to wrap conformally around near-cylindrical surfaces such as tree branches or pipelines (figure 1). This grapple is then attached to a cable and winch, allowing the robot to move relative to its perch with minimal power consumption.

To deploy this grapple in a mobile robot, a controller has been developed which allows the robot to reliably regulate tether tension during perching, and detect the attachment/detachment of the grapple with internal sensing alone. The control approach also enables a tethered flight mode allowing the robot to stably position itself relatively to the perch, and use the grapple as a releasable stability tether.

\section{Perching Control}

During perching the robot presented in this paper effectively tethers itself to a fixed surface, at which point the flight control must be adapted to account for the added force experienced from the attached tether. To ensure robust attachment, we have implemented a hybrid force/motion controller, to regulate interaction behaviour at low frequency and simultaneously control both cable tension and free motion. The grapple cable is deliberately built with some elasticity, such that the controller provides a direct force estimation.

Previous work on cable suspended aerial manipulation [13]-[18] has focused on the free flight of quadrotors with suspended payloads, without interaction with fixed structures. Additional work has explored the operation of tethered quadrotors [19]-[21]. As the perching system presented here requires direct interaction with the environment through a suspended cable as well as free flight we were obliged to take a different approach.

Here we introduce a modelling and control scheme which allows simultaneous switching between tethered and untethered modes without changing the control law.

\section{A. Dynamics formulation of quadrotor-grapple system}

We here model the dynamics of the quadrotor and grapple as two masses connected by a flexible cable (figure 2). While the model is simplified, it is nonetheless able to capture the key dynamics of the system, and is sufficient for accurate, reliable control. The dynamics of the combined system can be written as

$$
M \ddot{x}+G=N+U+F
$$

where $x:=\left[x_{Q} ; x_{G}\right] \in \Re^{6}$ is the configuration of the system with $x_{Q}, x_{G} \in \Re^{3}$ being the center of mass (CoM) locations of the quadrotor and of the grapple, respectively, $M:=\operatorname{diag}\left(m_{Q} I_{3 \times 3}, m_{G} I_{3 \times 3}\right) \in \Re^{6}$ is the inertia, and $G:=\left[-m_{Q} g e_{3} ;-m_{G} g e_{3}\right] \in \Re^{6}$ is gravity with $g \in \Re$ the gravitational acceleration and $e_{3}:=[0 ; 0 ; 1] \in \Re^{3}$.
The interaction between the quadrotor and the grapple is defined as an internal constrained force

$$
N=\left(\begin{array}{c}
r \\
-r
\end{array}\right) \rho
$$

where the tensile force $\rho \in \Re$ is determined as following

$$
\rho= \begin{cases}k_{c}(l-L)+b_{c} i, & \text { if } l \geq L \\ 0, & \text { otherwise, }\end{cases}
$$

where $k_{c}, b_{c}>0$ are the cable stiffness and damping coefficients, $l:=\left\|x_{G}-x_{Q}\right\|$ is the distance between the quadrotor and the grapple, and $L$ is the length of the cable at rest. The direction of the tensile interaction is

$$
r=\frac{1}{l}\left(x_{G}-x_{Q}\right) \in S^{2} \text {. }
$$

The external force $F$, is here assumed to be the interaction force which only occurs when the grapple attached to the perching surfaces. The grapple here is modelled as a point mass. The external force acting on the grapple is, therefore, aligned with the interaction direction $r$, such that,

$$
F=\left(\begin{array}{l}
0 \\
r
\end{array}\right) f
$$

with $f \in \Re$ being the magnitude of the external force and $f=0$ in free flight motion.

The control input $U \in \Re^{6}$ to the system is defined as

$$
U:=\left(\begin{array}{c}
-\lambda R e_{3} \\
0_{3 \times 3}
\end{array}\right)
$$

with $\lambda>0$ being the thrust of the quadrotor and $R \in S O$ (3) being the attitude of the quadrotor. The attitude of the quadrotor is generated by the following dynamics

$$
\begin{aligned}
& J_{Q} \dot{\omega}_{Q}=S\left(J_{Q} \omega_{Q}\right) \omega_{Q}+\tau \\
& \dot{R}=R S\left(\omega_{Q}\right)
\end{aligned}
$$

where $J_{Q} \in \Re^{6}$ is the inertia, $\omega_{Q} \in \mathfrak{s o}(3)$ is the angular velocity, $\tau \in \Re^{3}$ is the torque input of the quadrotor and $S(w) v=w \times v$ for any $w, v \in \Re^{3}$.

In our setup, the cable is attached close to the center of mass of the quadrotor. Thus, the attitude dynamics of the quadrotor is decoupled from the interaction of the tensile force. We here assume that the quadrotor attitude dynamics are much faster the translation dynamics and can be utilized to generate any desired thrust vector $\lambda R e_{3}$ for the system.

\section{B. Dynamics decomposition and control design}

We notice that the interaction between the quadrotor and the grapple/environment only effects the motion along the cable direction $r$ while motion in the remaining 5-DOF is unconstrained. This suggests separating the dynamics of the system into 5-DOF free motion space and 1-DOF interaction space for control. We here utilize the passive decomposition method [22] to decouple the dynamics while preserving the passivity of the system. By choosing the appropriate transformation, we can also separate the configuration of the system and reveal the mechanics of the perching motion. 
At each configuration $x=\left(x_{Q}, x_{G}\right) \in \Re^{6}$, we can decompose the tangent (or velocity) space $T_{x} \mathcal{M}=\Re^{6}$ and the cotangent (or force) space $T_{x}^{*} \mathcal{M}=\Re^{6}$ as following

$$
T_{q} \mathcal{M}=\Delta_{m} \oplus \Delta_{t}, \quad T_{q}^{*} \mathcal{M}=\Omega_{m} \oplus \Omega_{t}
$$

where $\Delta_{m}$ is the distribution of the null-space of $[r,-r] \in$ $\Re^{6}$ and $\Delta_{t}$ is the orthogonal complement of $\Delta_{m}$ with respect to the inertia metric of dynamics $M$; and $\Omega_{t}$ and $\Omega_{m}$ are the annihilating co-distributions of $\Delta_{t}$ and $\Delta_{m}$, respectively. In coordinates, we can write the velocity and the force as

$$
\dot{x}=\underbrace{\left[\begin{array}{cc}
\Delta_{m} & \Delta_{t}
\end{array}\right]}_{=: \Delta \in \Re^{6 \times 6}}\left[\begin{array}{c}
\nu_{m} \\
\nu_{t}
\end{array}\right], \quad U=\underbrace{\left[\begin{array}{cc}
\Omega_{m}^{T} & \Omega_{t}^{T}
\end{array}\right]}_{=: \Omega^{T} \in \Re^{6 \times 6}}\left[\begin{array}{c}
u_{m} \\
u_{t}
\end{array}\right] .
$$

where $\nu_{m}, u_{m} \in \Re^{5}$ are the transformed velocity and control components in the 5-DOF free motion space and and $\nu_{t}, u_{t} \in$ $\Re^{1}$ are those in the 1-DOF interaction (constrained) direction.

The coordinate expression of this decomposition is not unique. To facilitate the geometry presentation of the decomposition, we parameterize the interaction direction $r:=$ $[\cos \delta \cos \phi,-\sin \delta, \cos \delta \sin \phi]$ with $\Phi:=[\delta, \phi] \in \Re^{2}$ and choose

$$
\Delta=\left[\begin{array}{cc|c}
I_{3 \times 3} & \frac{-m_{G} l}{m_{G}+m_{Q}} \Gamma & \begin{array}{l}
\frac{-m_{G}}{m_{G}+m_{Q}} r \\
\underbrace{}_{3 \times 3} \frac{m_{Q} l}{m_{G}+m_{Q}} \Gamma
\end{array} \\
\Delta_{m} & \underbrace{\frac{m_{Q}}{m_{G}+m_{Q}}}_{\Delta_{t}} r
\end{array}\right] \in \Re^{6 \times 6},
$$

and $\Omega_{t}^{T}=[r,-r] \in \Re^{6 \times 1}, \Omega_{m}^{T}=M \Delta_{m}\left(\Delta_{m}^{T} M \Delta_{m}\right)^{-1} \in$ $\Re^{6 \times 5}$, where $\dot{r}=\Gamma \dot{\Phi}$ and

$$
\Gamma:=\left[\begin{array}{cc}
-\cos \phi \sin \delta & -\cos \delta \sin \phi \\
-\cos \delta & 0 \\
-\sin \delta \sin \phi & \cos \delta \cos \phi
\end{array}\right] \in \Re^{3 \times 2} .
$$

Note here that $r^{T} \Gamma=0$. We then can show that $\Delta_{t}^{T} M \Delta_{m}=$ $0_{1 \times 5}, \quad \Delta_{t} \Omega_{t}^{T}=1$.

With this choice of coordinate, we can then decompose not only the velocity but also the configuration, which is generally not always possible using passive decomposition. The configuration of the combined system can be separated as follows

$$
\begin{aligned}
& x_{Q}=x_{C}-\frac{m_{G} l}{m_{G}+m_{Q}} r \\
& x_{G}=x_{C}+\frac{m_{Q} l}{m_{G}+m_{Q}} r
\end{aligned}
$$

with $x_{C}:=\frac{1}{m_{G}+m_{Q}}\left(m_{Q} x_{Q}+m_{G} x_{G}\right) \in \Re^{3}$ being the center-of-mass of the quadrotor-grapple system. The geometry presentation of the the decomposed configuration is illustrated in Fig. 2. The velocity of the combined system can be rewritten as

$$
\left(\begin{array}{c}
\dot{x}_{G} \\
\dot{x}_{Q}
\end{array}\right)=\left[\begin{array}{ll}
\Delta_{m} & \Delta_{t}
\end{array}\right]\left(\begin{array}{c}
\dot{x}_{C} \\
\dot{\Phi} \\
\dot{l}
\end{array}\right) .
$$

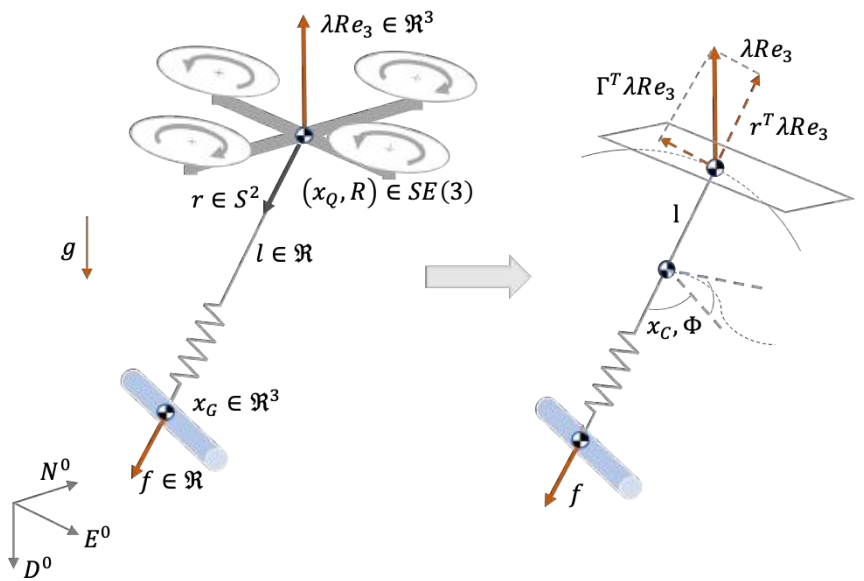

Fig. 2: The dynamics of the combined system $\left(x_{Q}, x_{G}\right) \in \Re^{6}$ is decomposed into 3-DOF translation dynamics (7) of the center-of-mass of the system, the 2-DOF rotation motion (8) of the system around the CoM and the 1-DOF interaction dynamics (9). The quadrotor thrust vector $\lambda R e_{3} \in \Re^{3}$ is the control input to the system with $\Gamma^{T} \lambda R e_{3} \in \Re^{2}$ being the motion control component and $r^{T} \lambda R e_{3} \in \Re$ the force control component.

Then, differentiating (6) with the above $\Delta$ and substituting them into (1), we obtain the decomposed dynamics

$$
\begin{aligned}
& \left(m_{Q}+m_{G}\right) \ddot{x}_{C}-\left(m_{Q}+m_{G}\right) g e_{3}=-\lambda R e_{3}+r f \\
& m_{Q} l \Gamma^{T} \Gamma \ddot{\Phi}+2 m_{Q} \Gamma^{T} \Gamma \dot{\Phi} \dot{\Phi}+m_{Q} l \Gamma^{T} \dot{\Gamma} \dot{\Phi}=\Gamma^{T} \lambda R e_{3} \\
& m_{Q} \ddot{l}+m_{Q} r^{T} \dot{\Gamma} \dot{\Phi} l+\frac{m_{G}+m_{G}}{m_{G}} \rho=r^{T} \lambda R e_{3}+\frac{m_{Q}}{m_{G}} f
\end{aligned}
$$

where (7) is the translation dynamics of the center-of-mass of the combined system, (8) is the free rotation motion of the system around the CoM, and (9) is the dynamics of the interaction between the quadrotor and the grapple. Note here that the sub-dynamics systems (7), (8) and (9) are decoupled and passive which is preserved under the passive decomposition [22]. With this property, the system will be stable under PD control law.

For the attitude dynamics (8), we use the following PD control law to stabilize the dynamics

$$
\Gamma^{T} \lambda R e_{3}:=-b_{\Phi} \dot{\Phi}-k_{\Phi}\left(\Phi-\Phi_{d}\right)
$$

where $\Phi_{d} \in \Re^{2}$ is the desired rotation angle and $b_{\Phi}, k_{\Phi}>0$ are the control gains. This control law ensures $\Phi \rightarrow \Phi_{d}$ as $t \rightarrow \infty$. To regulate interaction behavior, we use force control law

$$
r^{T} \lambda \operatorname{Re}_{3}:=\frac{m_{Q}+m_{G}}{m_{G}} \rho_{d}-k_{\rho} \int_{0}^{t}\left(\rho-\rho_{d}\right) d s
$$

with the desired tensile force $\rho_{d} \in \Re$ and control gain $k_{\rho}>0$. In practice, the feedback term $\rho$ is substituted by the estimated interaction force $\hat{\rho}$ using inertial measurements available on the quadrotor.

This illustrates an important property of the controller, that it does not require explicit knowledge of the cable length. This is important as it allows the system to adapt passively to 

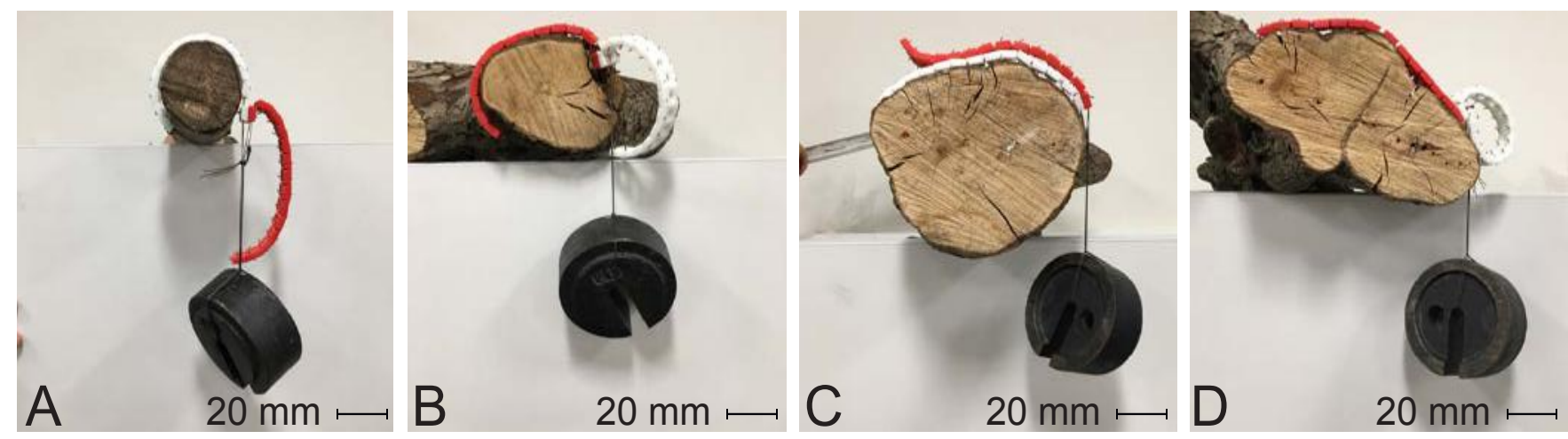

Fig. 3: Grapple shown suspending a $2 \mathrm{~kg}$ payload from a variety of branch shapes, and conforming to irregular geometries.

the unpredicted shape of the perching target (figure 3), and the difficulty in predicting the location at which the grapple binds to a perching target. This also allows the tether length to be freely adjusted by a winch, for more manoeuvrability. It is reasonable to assume that the external force $f$ and the desired motion/force $\Phi_{d}, \rho_{d}$ are all bounded, which ensures the stability of the internal dynamics (7).

This designed hybrid force/motion control is then decoded into the control inputs $(\lambda, \tau)$ of the quadrotor as follows: 1) compute the desired thrust vector $\lambda R e_{3}$ from the control (10) and $(11) ; 2$ ) given desired thrust vector, calculate the throttle and roll, pitch, yaw commands for the quadrotor as shown in [23]; and 3) desired throttle and attitude commands then generated by thrust $\lambda \in \Re$ and control input $\tau \in \Re^{3}$ in the low-level attitude control of the quadrotor.

\section{MeChaniCAL DeSIGN}

\section{A. Compliant Grapple System}

The grapple is formed from individual plastic links, with a trapezoidal cross section (figure 4A). Each link slides freely along a flat cable through its centre, with the exception of the final, furthest link, to which the cable is attached. The faces of each segment are angled, such that when a shear force is applied to a segment, the cable tension causes the grapple to curl. This ensures that the grapple makes contact with the entire surface, and will reliably grip a branch or pipe completely passively. The angle the faces make, $\phi$, and the length of each segment, $L$, determines the minimum diameter of branch, $D=L \tan (\phi / 2)$, that the grapple can curl around. The grapple configuration presented in this paper uses 14 segments, each with a $20 \mathrm{~mm}$ length and a face angle of $20^{\circ}$.

The grapple is sufficiently wide that it will roll onto the spined face where it impacts a perch on its side, but because the grapple curls only in one direction, grapples are used in pairs, attached at the first link with a common tether, such that attachment is possible from any direction (figure 4B). For attachment to trees, each link has a pair of sharpened steel spines protruding from the underside (figure 4C), formed from $0.8 \mathrm{~mm}$ diameter spring steel (ASTM A228). These spines provide attachment to rough or soft surfaces, where the spines can gain sufficient purchase. For smooth ferrous surfaces, the grapple can also be configured with magnets, and perch using contact friction on the links (figure 4D).

Each segment uses a pair of spines with the points aligned parallel to the curling axis of the grapple. This means that individual segments can pivot around the point at which the spines make contact, and the grapple can conform to the surface more easily (figure 3). Adding additional spines to a segment would not allow more load to be supported per segment unless individual spines were permitted to move and bring all spines into contact with the surface, as in the devices presented in [24], but the additional complexity was not warranted. The grapple has a total of 56 spines, although only 28 spines can be used at once. This is comparable to a previous perching UAV using spines for a vertical surface, which used 10 spines to support a $0.4 \mathrm{~kg}$ load [25] or a hexapedal climbing robot which used 192 spines to support a $3.8 \mathrm{~kg}$ load [24]. In this instance, the curling of the grapple and the use of a force controller ensures that the spines are favourably loaded with tangential forces, and the additional spines are not required to sustain the loads encountered during perching. Although the links are identical and the grapple's maximum curvature is constant, because the indivual links of the grapple can move slightly relative to one another the grapple is able to conform to the shape of perches which are convex but not cylindrical (figure 3D).

The compliance ensures robust perching to surfaces, but when tension is removed from the grapple, each individual segment can be easily detached from the substrate in an unpeeling motion, with segments being detached one by one with far lower force. The grapple is thereby able to sustain significant force $(>60 \mathrm{~kg}$, depending on substrate, see section IV-A) while still being passively detachable using light propeller thrust.

\section{B. Integrated Perching Platform}

The grapple is integrated into a quadrotor airframe (DJI F450) with a companion computer (Intel NUC) for trajectory generation and a Pixhawk 4 flight controller running PX4 (table II). The grapple is attached to the base of the UAV via a motorised winch which allows the grapple to be extended and retracted and the robot to move up and down from a perch. 

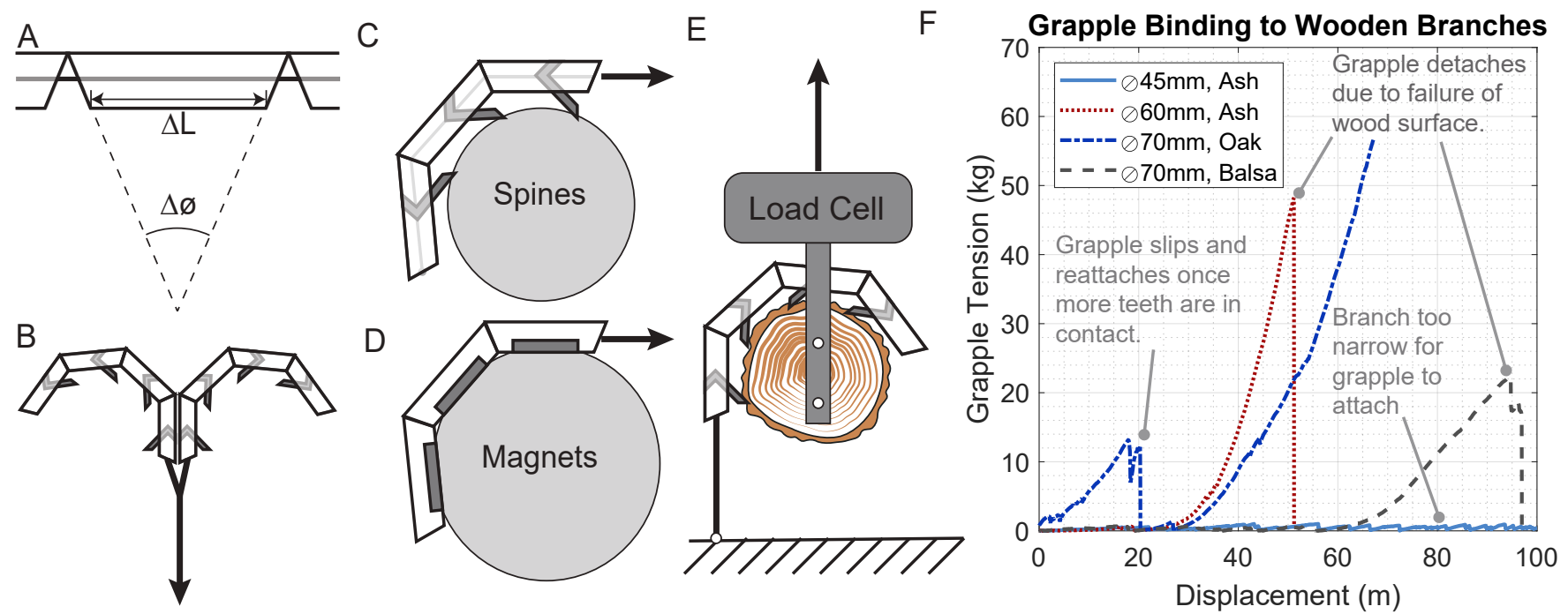

Fig. 4: A: Grapple schematic, showing the central tether, and key dimensions. B: Paired grapples, used to ensure passive attachment regardless of approach direction. C: Grapple configured with microspines, illustrating minimum effective perch radius. D: Grapple configured with magnets for attachment to ferrous perches. E: Grapple tension test layout schematic, showing test direction. F: Grapple tension test results on a variety of branches. It can be seen in some tests that the grapple slips slightly before fully engaging, as initially only a few microspines make contact with the branch. The UAV used in this paper weighs $1.7 \mathrm{~kg}$, so the $60 \mathrm{~kg}$ capacity tested here is far more than sufficient.

\begin{tabular}{|l|c|r|}
\hline Component & Mass (grams) & /Total \\
\hline Quadrotor Airframe & 866 & $47 \%$ \\
Battery (4S 2200mAh) & 218 & $12 \%$ \\
Computing (NUC) & 602 & $33 \%$ \\
Winch System & 108 & $6 \%$ \\
Grapple & 32 & $2 \%$ \\
\hline Total & $\mathbf{1 7 6 6}$ & $\mathbf{1 0 0} \%$ \\
\hline
\end{tabular}

TABLE I: Mass breakdown for the Perching UAV (configured with microspines). The grapple is only $2 \%$ of the total airframe weight, and the winch system $6 \%$, leaving significant space for additional payload.

\begin{tabular}{|l|r|l|}
\hline Item & Dimension & units \\
\hline Quadrotor Wheelbase & 450 & $\mathrm{~mm}$ \\
Grapple segment width & 20 & $\mathrm{~mm}$ \\
Grapple segment thickness & 6 & $\mathrm{~mm}$ (per side) \\
Grapple segment count & 14 & (per side) \\
Grapple total length & 196 & $\mathrm{~mm}$ \\
\hline
\end{tabular}

TABLE II: Key dimensions for the robot and grapple.

The grapple is attached to a kevlar cable, connected via a $30 \mathrm{~cm}$ section of elastic cable. The compliance introduced by the elastic section improves perching performance when attaching the grapple to a substrate. The controller is able to perch and unperch the robot in any orientation, so the grapple can be mounted on either the top or bottom of the drone, depending on other payload considerations. The grapple was also tested on a larger drone with a sensor payload below, which necessitated mounting the cable on the top, and the inclusion of a cable guide to prevent entanglement with the rotors (figure 5B).

\section{EXPERIMENTS}

\section{A. Grapple Tension Testing}

To evaluate its load carrying capacity, the grapple was tested on several different tree branches using a Instron tension testing machine. During testing, the grapple was placed over the test branch such that it hung below, not in contact, and was then dragged over the branch by the test machine at a speed of $50 \mathrm{mms}^{-1}$ until shear on the microspines caused it to engage (figure 4E). Results for different sample perches types are shown in figure $4 \mathrm{~F}$.

It was found that the grapple was limited not by the strength of the microspines, but by the strength of the substrate to which is was attached. However, even extremely soft wood such as balsa showed a tension capacity far in excess of any reasonable weight for a UAV (an order of magnitude greater than the weight of the platform used in this paper) and it can be reasonably concluded that the grapple load capacity will not be limiting.

\section{B. Perching Experiments}

The grapple was integrated with a small multirotor UAV weighing 1766 grams (table II), and a motorised winch system to allow movement on the perch. The perching sequence was tested on a variety of substrates, including tree branches (figure 5A) and steel pipe (figure 5B). The internal tension estimation on the UAV is shown in figure 5C, showing the UAV identifying that the grapple has attached as it approaches the branch and the force control used to ensure that tension is maintained as the UAV moves smoothly to a perched state. Figure 5C also shows the ease with which the grapple releases from the branch after perching. Figure 5D 


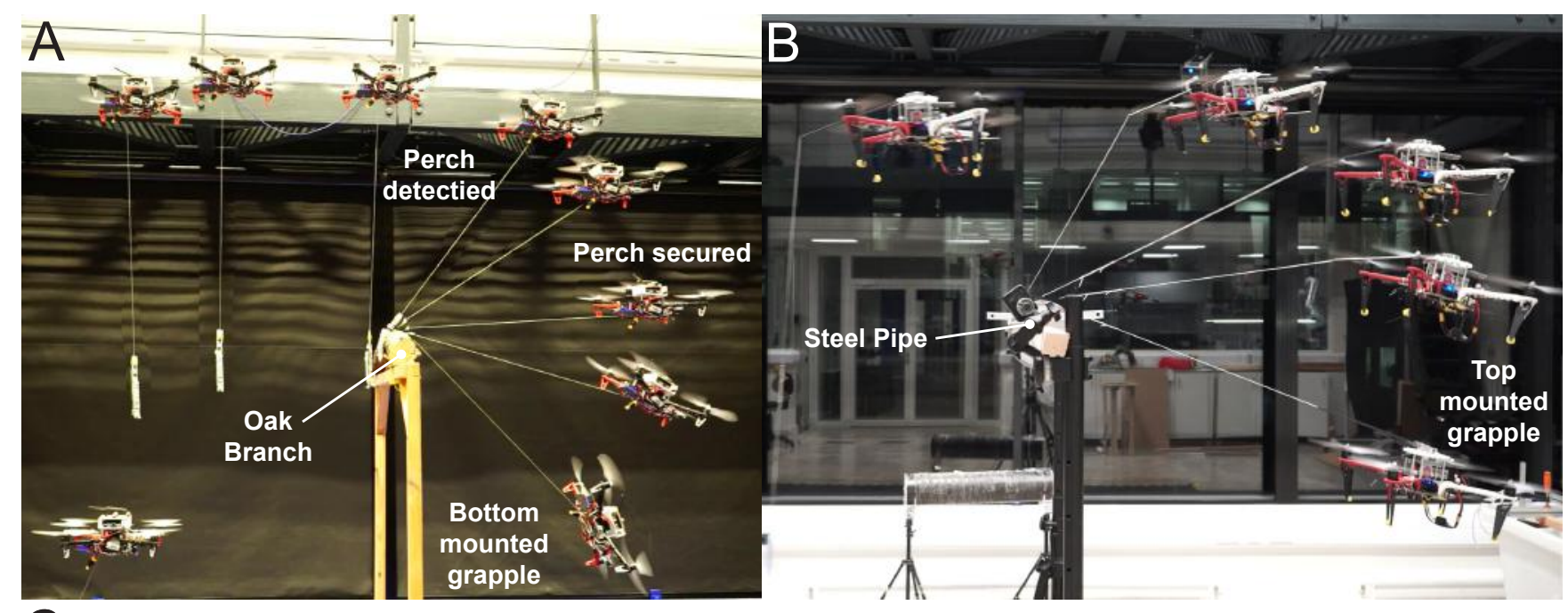

C
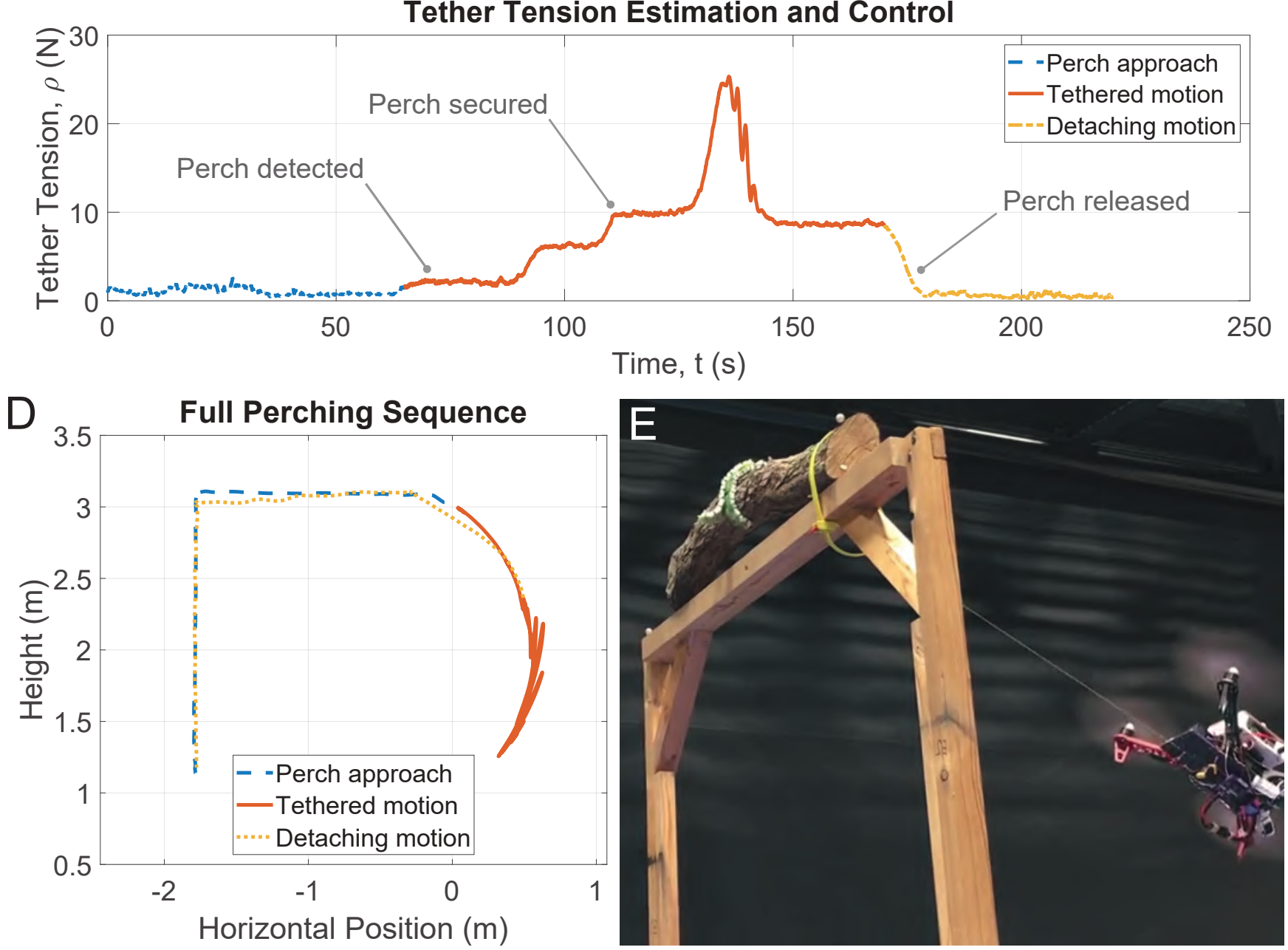

Fig. 5: A: Perching sequence on an ash branch with microspines, showing attachment and transition to a stable perch. B: Perching sequence on a steel pipe, with magnets. The grapple can be attached to the bottom or top of the drone, depending on payload configuration. Here, with the cable on the top, a rigid cable guide tube is used to keep the tether away from the props C: Time history of internal tether tension estimation during a perch sequence with microspines. The plot shows the detection of grapple attachment and force control during perching, as well as the ease with which the grapple is released. D: Trajectory time history from a the same perch manoeuvre. E: Close up of an attached UAV, showing the grapple passively wrapped to the perch and the UAV stably holding its orientation. 

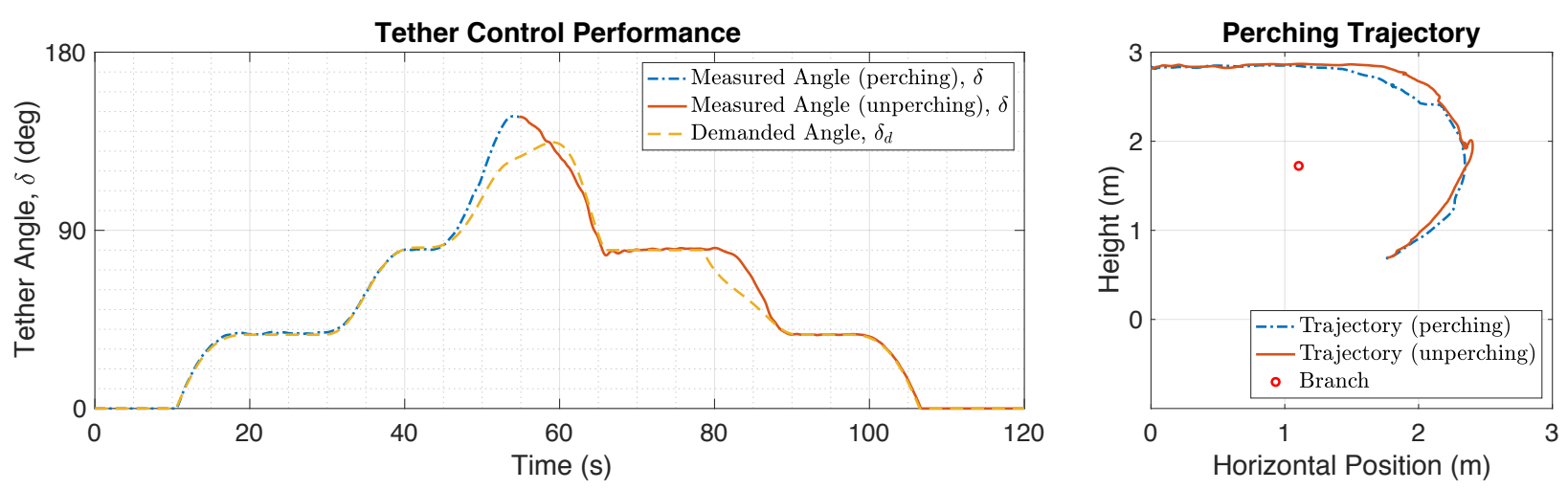

Fig. 6: Test showing the control fidelity during a perch/unperch manoeuvre. The actual position is recorded using a Vicon motion capture system. A: Demanded/actual tether angle. B: Resulting trajectory, with branch location indicated. The results show that the controller is functioning effectively, in spite of the unpredictable attachment of the grapple.

shows the trajectory followed by the UAV as it perches and unperches from the branch.

Once attached the the branch the controlled can hold the $\mathrm{UAV}$ at any orientation, by using the grapple as a tether. Figure 5E shows the grapple bound to the branch and the UAV holding a non-equilibrium position using tether tension and propeller thrust.

Testes were also repeated using a magnetic grapple configuration (see section III-A). In this configuration, the grapple tension is less significant, and perching was reliable, provided a rubber coating was used to prevent the magnets from fracturing.

The performance of the controller was measured by comparing demanded angle with motion capture data during a perch and unperch manoeuvre (figure 6). The controller was found to conform accurately to the position demands, and to respond effectively when the command angle was changed as the perch sequence progressed.

Finally, the grapple was tested outside on a variety of natural perches (figure 1A). Anecdotally, the grapple was found to be very reliable when attaching to rough-barked tree species such as oak or willow, but that perching could fail if the attachment process proceeded too quickly on smooth, hard tress such as hazel.

\section{CONClusions ANd Future Work}

In this paper we have demonstrated a perching robot which uses a specialised flight controller which exploits the compliance of a passive grapple for robust, reliable attachment to various substrates. We use a control approach which is independent of tether length, allowing tensile perching under unpredictable conditions or perch geometry. This robot could find application in many inspection and observation tasks, where access with terrestrial robots is limited and the flight times of aerial robots is restrictive. The robot is also able to use its perching system as a detachable tether, which allows it to maintain a position/orientation with improved stability.
This potentially could be used as a stability/safety measure in wind or other destabilising flight conditions.

Future work will focus on the integration of sensing systems to allow autonomous identification of perches, including assessment of rugosity, which is important to the effectiveness of the grapple when configured with spines. We will also explore applications of the passive-adaptive grapple system to other aerial manipulation and grasping applications.

The grapple presented here weighs only 32 grams, but is capable of supporting loads far in excess of typical flying robot weights and could potentially be used in other, far heavier robots. The controller used to attach the grapple would also be an effective system for tethered robots and suspended loads. The tensile anchoring system and controller also has utility in providing stable, precise navigation where significant external perturbations are present, which will be explored in future work.

\section{ACKNOWLEDGEMENTS}

This work was partially supported by research awards from the Engineering and Physical Sciences Research Council (EPSRC) under grant agreements EP/N018494/1, EP/R026173/1, EP/R009953/1 and EP/R02572X/1. Mirko Kovač is supported by the Royal Society Wolfson Fellowship under grant agreement RSFR1180003.

\section{REFERENCES}

[1] D. Floreano and R. J. Wood, "Science, technology and the future of small autonomous drones," Nature, vol. 521, no. 7553, p. 460, 2015.

[2] M. Kovac, "Learning from nature how to land aerial robots," Science, vol. 352, no. 6288, pp. 895-896, 5 2016. [Online]. Available: http://www.sciencemag.org/cgi/doi/10.1126/science.aaf6605

[3] P. Sareh, P. Chermprayong, M. Emmanuelli, H. Nadeem, and M. Kovac, "Rotorigami: A rotary origami protective system for robotic rotorcraft," Science Robotics, vol. 3, no. 22, p. eaah5228, 2018.

[4] L. Daler, A. Klaptocz, A. Briod, M. Sitti, and D. Floreano, "A perching mechanism for flying robots using a fibre-based adhesive," in Robotics and Automation (ICRA), 2013 IEEE International Conference on. IEEE, 2013, pp. 4433-4438. 
[5] W. Chi, K. Low, K. H. Hoon, and J. Tang, "An optimized perching mechanism for autonomous perching with a quadrotor," in Robotics and Automation (ICRA), 2014 IEEE International Conference on. IEEE, 2014, pp. 3109-3115.

[6] A. Braithwaite, T. Alhinai, M. Haas-Heger, E. McFarlane, and M. Kovač, "Tensile web construction and perching with nano aerial vehicles," in Robotics Research. Springer, 2018, pp. 71-88.

[7] M. A. Estrada, S. Mintchev, D. L. Christensen, M. R. Cutkosky, and D. Floreano, "Forceful manipulation with micro air vehicles," Science Robotics, vol. 3, no. 23, 2018. [Online]. Available: http://robotics.sciencemag.org/content/3/23/eaau6903

[8] M. Kovač, J. Germann, C. Hürzeler, R. Y. Siegwart, and D. Floreano, "A perching mechanism for micro aerial vehicles," Journal of MicroNano Mechatronics, vol. 5, no. 3-4, pp. 77-91, 12 2009. [Online]. Available: http://link.springer.com/10.1007/s12213-010-0026-1

[9] S. Kim, A. T. Asbeck, M. R. Cutkosky, and W. R. Provancher, "Spinybotii: climbing hard walls with compliant microspines," in Advanced Robotics, 2005. ICAR'05. Proceedings., 12th International Conference on. IEEE, 2005, pp. 601-606.

[10] W. R. T. Roderick, M. R. Cutkosky, and D. Lentink, "Touchdown to take-off: at the interface of flight and surface locomotion," Interface Focus, vol. 7, no. 1, p. 20160094, 2 2017. [Online]. Available: http://rsfs.royalsocietypublishing.org/lookup/doi/10.1098/rsfs.2016.0094

[11] A. Parness, M. Frost, N. Thatte, J. P. King, K. Witkoe, M. Nevarez, M. Garrett, H. Aghazarian, and B. Kennedy, "Gravity-independent rock-climbing robot and a sample acquisition tool with microspine grippers," Journal of Field Robotics, vol. 30, no. 6, pp. 897-915, 2013.

[12] J. Molina and S. Hirai, "Aerial pruning mechanism, initial real environment test," Robotics and biomimetics, vol. 4, no. 1, p. 15, 2017.

[13] I. Palunko, R. Fierro, and P. Cruz, "Trajectory generation for swingfree maneuvers of a quadrotor with suspended payload: A dynamic programming approach," in IEEE International Conference on Robotics and Automation, 2012, pp. 2691-2697.

[14] K. Sreenath, T. Lee, and V. Kumar, "Geometric control and dierential atness of a quadrotor uav with a cable-suspended load," in IEEE Conference on Decision and Controlon Decision and Control, 2013, pp. 2269-2274.

[15] F. A. Goodarzi, D. Lee, and T. Lee, "Geometric stabilization of a quadrotor UAV with a payload connected by flexible cable," in American Control Conference, 2014, pp. 4925-4930.

[16] P. Kotaru, G. Wu, and K. Sreenath, "Dynamics and control of a quadrotor with a payload suspended through an elastic cable," in American Control Conference, 2017, pp. 3906-3913.

[17] P. Foehn, D. Falanga, N. Kuppuswamy, R. Tedrake, and D. Scaramuzza, "Fast Trajectory Optimization for Agile Quadrotor Maneuvers with a Cable-Suspended Payload," in Robotics: Science and Systems, 2017.

[18] S. Tang, V. Wueest, and V. Kumar, "Aggressive Flight With Suspended Payloads Using Vision-based Control," IEEE Robotics and Automation Letters, vol. 3, no. 2, pp. 1152-1159, 2018.

[19] M. Tognon, A. Testa, E. Rossi, and A. Franchi, "Takeoff and landing on slopes via inclined hovering with a tethered aerial robot," in IEEE International Conference on Intelligent Robots and Systems, 2016, pp. $1702-1707$.

[20] M. M. Nicotra, R. Naldi, and E. Garone, "Nonlinear control of a tethered UAV: The taut cable case," Automatica, vol. 78, pp. 174-184, 2017.

[21] M. Tognon and A. Franchi, "Dynamics, control, and estimation for aerial robots tethered by cables or bars," IEEE Transactions on Robotics, vol. 33, no. 4, pp. 834-845, 2017.

[22] D. Lee and P. Y. Li, "Passive decomposition of mechanical systems with coordination requirement," IEEE Transactions on Automatic Control, vol. 58, no. 1, pp. 230-235, 2013.

[23] H.-N. Nguyen, S. Park, J. Park, and D. Lee, "A novel robotic platform for aerial manipulation using quadrotors as rotating thrust generators," IEEE Transactions on Robotics, vol. 34, no. 2, pp. 353-369, 2018.

[24] A. T. Asbeck and M. R. Cutkosky, "Designing compliant spine mechanisms for climbing," Journal of Mechanisms and Robotics, vol. 4, no. 3, p. 031007, 2012.

[25] A. Lussier Desbiens, A. T. Asbeck, and M. R. Cutkosky, "Landing, perching and taking off from vertical surfaces," The International Journal of Robotics Research, vol. 30, no. 3, pp. 355-370, 2011. 